\title{
Optimization and comparison between two 6-DoF parallel kinematic machines for HIL simulations in wind tunnel
}

\author{
Enrico Fiore and Hermes Giberti
}

Politecnico di Milano, Mechanical Department Via La Masa 1, 20156 Milano, Italy

\begin{abstract}
Parallel Robots are for sure the best choice for those applications in which a high accuracy and a high stiffness are required. As a matter of fact the closed-loop kinematic chains that characterize these machines guarantee a very low sensitivity of the end-effector position to the various sources of error. However the working volume of parallel kinematic machines is in general limited with respect to their dimensions. Moreover the reduced mobility range of the passive joints represents a critical aspect that cannot be underestimated. For these reasons PKMs should be optimized for the application they are meant.Given the specifications of the workspace that the machine should be able to cover, it's necessary to identify an architecture that is suitable for this specific task. This paper describes the optimization process and the comparison between two candidates, the Hexaslide and the Gough-Stewart platform, in order to find out which one is the best choice for a task that requires to reproduce the hydrodynamic effect of sea waves at the bottom of scale models of off-shore wind turbines and boats during HIL simulations in the wind tunnel of the Politecnico di Milano.
\end{abstract}

\section{Introduction}

Nowadays one of the most important lines of research is the study of the interaction between the wind and civil structures. However in some cases the wind is not the only source of dynamic excitation, for example on sailing boats and on floating off-shore wind turbines (FOWT) the contribution given by the sea cannot be neglected.

The experimental phase is fundamental to understand the aerodynamic and hydrodynamic phenomena that are involved: as a matter of fact by properly setting the control parameters of the inclination of the blades of wind turbines it's possible to have a better exploitation of the wind energy; instead as regards the sailboats it's important to understand which is the fluid-structure interaction during the change of direction.

Three kinds of experiment can be carried out: wind tunnel tests; tank tests; full scale simulations. Moreover, through these tests it is possible to validate CFD codes and to set all the parameters needed for the numerical simulations. As regards the wind tunnel tests, the real wind profile can be reproduced with a good approximation unlike the hydrodynamic effect of the sea waves. For this reason it's important to equip the wind tunnel of the Politecnico di Milano with a machine that takes as a input the forces and moments recorded by a 6axes balance at the bottom of the scale models of FOWTs and boats, and on the basis of a mathematical model of the sea imposes a movement to the scale models [1].

In the beginning the hypothesis of using a serial robot was considered. Indeed these manipulators are definitely the most diffused commercially speaking and moreover don't suffer any problem in terms of workspace dimensions. However in serial manipulators the errors of each joint add up to those of the successive joints with the result of worsening the accuracy of the positioning of the end-effector. Due to their intrinsic embossed structure they have to bear with high bending loads and so the links that connect one actuated joint to the other become increasingly bulky starting from the end-effector towards the base joint. At this point the parallel kinematic manipulators (PKM) are taken into account. The performances of these machines strictly depend on their dimensions and so it becomes difficult to make a comparison between different architectures as stated by Merlet in [2]. However it's still possible to highlight their general peculiarities. For sure their working volume is lower than that of serial robots, but these machine are characterized by a high stiffness thanks to the fair distribution among the links of the loads acting on the end-effector. Furthermore in some PKMs the joints at the ends of the links are spherical or universal and so the loads the links are subject to are exclusively of axial nature. In parallel kinematic manipulators the interaction mechanisms between the errors of each joint and those of the end-effector are far too complex with respect to serial robots with the result of having a lower sensitivity of the positioning errors of the end-effector.

After these considerations two different architectures of hexapods were identified as suitable candidates to perform the hardware in the loop (HIL) simulations: (1) the Hexaslide which is made of a mobile platform 
connected to six linear guides by means of six links of fixed length, so that six independent kinematic chains can be identified belonging to the PUS family. A detailed description of 6-DoF PUS PKMs is provided by Bonev in [3]. The actuating system of the Hexaslide is fixed to the ground so that the resulting moving mass is represented just by the slender links and the thin platform. However this machine is characterized by a great footprint. (2) the second candidate is the well-known Gough-Stewart platform. This machine is made of six links of variable length that connect the mobile platform to six universal joints fixed to the ground. In this case the kinematic chains belong to the UPS family. Despite the lower dimensions with respect to the Hexaslide architecture, the actuation in this manipulator is provided directly by the links. Their length is changed by means of a ball-screw transmission unit which is characterized by a very high inertia. Furthermore the motors cannot be fixed to the ground but should be mounted on the links and so they contribute to increase the total moving mass.

The vertical available space for the machine in the wind tunnel is limited by the boundary layer in the zone near the ceiling. As a matter of fact here the wind profile is different with respect to the real one and so it's important that the scale models don't enter in this zone. For this reason the tool center point (TCP) of the manipulator should stay as low as possible. The two chosen architectures allow to have a very low TCP and in addition they avoid the risk of self collision between the machine elements which in general is a critical aspect of PKMs.

In order to respect the requirements in terms of working space and of constraints, the manipulator should undergo an optimization process. For both the Hexaslide and the Gough-Stewart a genetic algorithm was used to optimize the two machines.

The following sections are organized as follow: Section 2 describes the inverse kinematics of the GoughStewart platform and of the Hexaslide; Section 3 provides a description of the technical specifications and a detailed explanation of the cost function and of the constraints; numerical results and the comparison between the two architectures are presented in Section 4.

\section{Inverse kinematics}

In order to bring the robot mobile platform to the desired poses it is fundamental to establish the relationships between the TCP coordinates and the joint space ones. In general for PKMs it's easier to solve the inverse kinematics [4], that is the computation of the joint space coordinates once the TCP coordinates are known. The solution of the inverse kinematics requires to use two different reference systems, a global one denoted by unit vectors $\widehat{\boldsymbol{x}}, \widehat{\boldsymbol{y}}, \hat{\boldsymbol{z}}$, and a relative one attached to the mobile platform and denoted by unit vectors $\widehat{\boldsymbol{x}}^{\prime}, \widehat{\boldsymbol{y}}^{\prime}, \hat{\mathbf{z}}^{\prime}$.

\subsection{Gough-Stewart Platform}

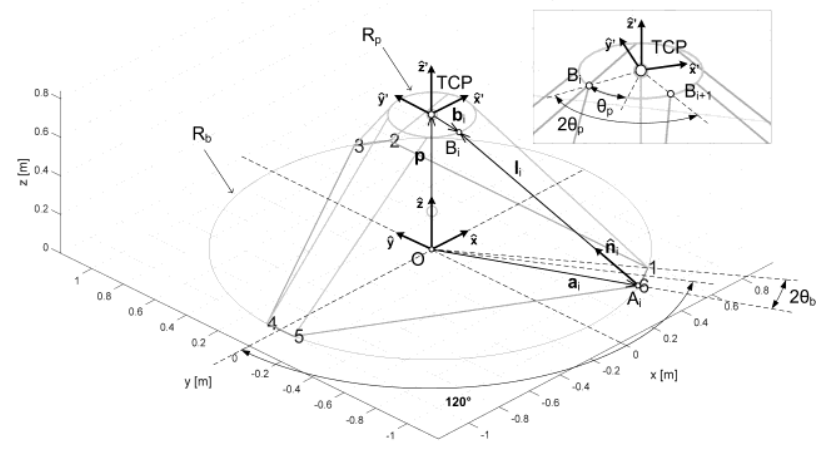

Figure 1. Gough-Stewart platform i-th kinematic chain.

The adopted strategy consists in closing a kinematic chain in order to determine the position of the platform joints with respect to the base joints. Once the coordinates of the $i$-th platform joint are known it is possible to compute the length of the $i$-th link. Looking at Figure 1 it is possible to write:

$\boldsymbol{l}_{i}=\boldsymbol{p}+[R] \boldsymbol{b}_{i}^{\prime}-\boldsymbol{a}_{i}$

where $\boldsymbol{l}_{i}$ is the vector aligned with the $i$-th link, $\boldsymbol{p}$ is the vector containing the position and orientation of the mobile platform, [R] is the rotation matrix, $\boldsymbol{b}^{\prime}{ }_{i}$ expresses the position of the $i$-th platform joint with respect to the TCP in the relative frame and $\boldsymbol{a}_{i}$ is the is the vector that describes the position of the $i$-th base joint. By squaring equation (1) one gets:

$l_{i}^{2}=\boldsymbol{p}^{T} \boldsymbol{p}+\boldsymbol{b}_{i}^{\prime}{ }^{T} \boldsymbol{b}^{\prime}{ }_{i}+\boldsymbol{a}_{i}{ }^{T} \boldsymbol{a}_{i}+2 \boldsymbol{p}^{T}[R] \boldsymbol{b}^{\prime}{ }_{i}-2 \boldsymbol{p}^{T} \boldsymbol{a}_{i}-$

$2 \boldsymbol{a}_{i}^{T}[R] \boldsymbol{b}^{\prime}{ }_{i}$

\subsection{Hexaslide}

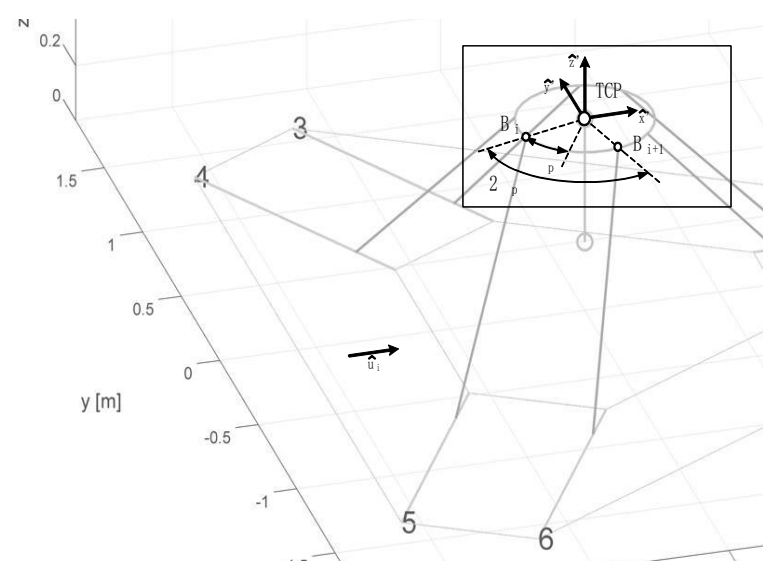

Figure 2. Hexaslide i-th kinematic chain.

For the Hexaslide a similar procedure can be used. Anyway in this case it's necessary to use two kinematic chains, the first one to find the position of the $i$-th platform joint with respect to the $i$-th guide and the latter to compute the position of the $i$-th slider on the relative guide.

So with reference to Figure 2 it is possible to write: 
$\boldsymbol{l}_{i}=\boldsymbol{d}_{\boldsymbol{i}}+q_{i} \widehat{\boldsymbol{u}}_{i}$ with $\boldsymbol{d}_{\boldsymbol{i}}=\boldsymbol{p}+[R] \boldsymbol{b}_{i}^{\prime}-\boldsymbol{s}_{i}$

where $\widehat{\boldsymbol{u}}_{i}$ is a unitary vector aligned with the $i$-th guide axis, $\boldsymbol{s}_{i}$ is the position of the same guide with respect to the fixed frame, while $q_{i}$ represents the position of the $i$-th slider along the corresponding guide axis.

After some simple mathematical passages the following expression is found:

$q_{i}=\boldsymbol{d}_{i}^{T} \widehat{\boldsymbol{u}}_{i} \pm \sqrt{\boldsymbol{d}_{i}^{T}\left(\widehat{\boldsymbol{u}}_{i} \widehat{\boldsymbol{u}}_{i}^{T}-[I]\right) \boldsymbol{d}_{i}+l_{i}^{2}}$

\subsection{Velocity Analysis}

Once solved the inverse kinematics it is possible to find the relationship between the sliders/links velocity and the velocity of the TCP. For the Gough-Stewart it can be shown that for the $i$-th kinematic chain the following expression holds:

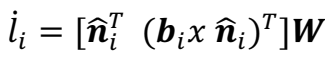

where $\boldsymbol{W}=\left[\dot{x}, \dot{y}, \dot{z}, \omega_{x}, \omega_{y}, \omega_{z}\right]^{T}$ is a vector containing both the translational and angular components of TCP velocity and $\widehat{\boldsymbol{n}}_{\boldsymbol{i}}$ is the $i$-th link unitary vector.

For the Hexaslide a similar expression is obtained:

$\widehat{\boldsymbol{n}}_{i}^{T} \widehat{\boldsymbol{u}}_{i} \dot{q}_{i}=\left[\widehat{\boldsymbol{n}}_{i}^{T}\left(\boldsymbol{b}_{i} x \widehat{\boldsymbol{n}}_{i}\right)^{T}\right] \boldsymbol{W}$

Considering all the six links the previous expressions can be recast in a matrix form using the Jacobian matrix $[J]$ :

$\boldsymbol{W}=[J] \dot{\boldsymbol{q}}$

\subsection{Kinetostatic}

The Jacobian matrix is fundamental also for the kinetostatic problem. As a matter of fact by applying the virtual work principle it's possible to compute the actuators forces $\boldsymbol{\tau}_{\text {act }}$ needed to equilibrate the external forces and moments acting on the TCP $\boldsymbol{f}_{e, T C P}$ :

$\delta \boldsymbol{X}^{T} \boldsymbol{f}_{e, T C P}+\delta \boldsymbol{q}^{T} \boldsymbol{\tau}_{a c t}=0$

It is possible to recast the virtual work principle as follows:

$\delta \boldsymbol{q}^{T}\left([J]^{T} \boldsymbol{f}_{e, T C P}+\boldsymbol{\tau}_{a c t}\right)=0$

Since this expression must hold for all virtual variation of the joint coordinates the following expression is found:

$\boldsymbol{\tau}_{a c t}=-[J]^{T} \boldsymbol{f}_{e, T C P}$

If the aim is to study how the forces acting on the TCP are transformed in those acting on the sliders along the guide axes it's sufficient to change the sign of the previous expression. In this sense the transposed Jacobian matrix assumes the meaning of a generalized transmission ratio. The vector $\boldsymbol{f}_{\boldsymbol{e}, \boldsymbol{T C P}}$ is composed of three elements representing the external forces and three elements representing the external moments acting on the TCP. In order to normalize all the terms of the jacobian matrix and to consider the isotropy of the machine it's necessary to use a scale factor defined as characteristic length. The study of the isotropy of PKMs depending on their design parameters is a field in which many researchers are involved $[5,6]$. The characteristic length is defined in many ways in literature, in this paper the definition provided by Fassi and Legnani in [7] is used. This parameter allows to define a normalized Jacobian matrix which is used for the formulation of the kinetostatic constraint and is denoted as $\left[J_{n}\right]$.

\section{Optimization}

The optimization process requires to define a set of variables that constitute the quantities that can be varied in order to achieve a specific goal, in particular the minimization of a cost function. In this case as often happens in the optimization problems, the system to be optimized is subject to a set of constraints of various nature. The algorithm used to carry out the optimization is a genetic algorithm. Thanks to the symmetry of the two machines it is possible to use a single objective algorithm; on the contrary the optimization process of other PKMs may require a multi-objective algorithm. An example of a multi-objective genetic algorithm is presented in $[8,9]$. In this paragraph the technical specifications, the cost function, the parameterization of the two manipulators and the constraints are described.

\subsection{Technical Specifications}

The researchers of the wind tunnel of the Politecnico di Milano studied the scaling of the aerodynamic models and the movements of the real FOWTs and boats. These studies allowed to define the workspace the TCP should be able to move in. The workspace is represented by a parallelepiped of dimensions $300 \mathrm{~mm} \times 150 \mathrm{~mm} \times 150$ $\mathrm{mm}$ within which the TCP can assume any combination of the rotations described. Due to the problems related to the limited vertical space, the center of the workspace is set to be in the point of coordinates $[0,0,0.7]^{T}$.

\subsection{Cost Function}

The cost function for an individual characterized by a certain set of variables consists in the computation of the portion of volume of the desired workspace (WSd) that is not possible to cover. To this end the WSd is divided into sub-volumes using a three dimensional grid. Given the dimensions of the WSd and chosen a number of points $N_{\text {grid }}$ on each edge, the WSd is divided into $N_{\text {grid }}^{3}$ subportions whose volume is equal to:

$\Delta v=\frac{2 \Delta x \times 2 \Delta y \times 2 \Delta z}{N_{\text {grid }}^{3}}$

For a fixed orientation $\overline{\boldsymbol{\Theta}}=[\bar{\alpha}, \bar{\beta}, \bar{\gamma}]^{T}$ the not covered volume is computed. In other words it's necessary to 
assess how many points don't comply with all the imposed constraints:

$v_{n c}(\overline{\boldsymbol{\Theta}})=\sum_{i} k_{i} \Delta v$

where $k_{i}$ is equal to 1 if at least one constraints is violated and 0 otherwise. The procedure is repeated for each combination of the angular parameters $\alpha, \beta, \gamma$ and the total not covered volume is computed as:

$V_{n c}=\sum_{j} v_{n c}\left(\overline{\boldsymbol{\Theta}}_{j}\right)$

\subsection{Constraints}

It's important to guarantee that the kinematics of the manipulator is respected, to avoid the self-collision between the elements of the machine and that the force transmitted to the motors is not too high. For these reasons the following constraints are imposed:

I. Kinematic constraints: this kind of constraints guarantee to cover each point of the WSd:

i. $\quad \Delta_{i} \geq 0$ (Hexaslide only): for each link it's necessary to assess that $\Delta_{i}=\boldsymbol{d}_{i}^{T}\left(\widehat{\boldsymbol{u}}_{i} \widehat{\boldsymbol{u}}_{i}^{T}-[I]\right) \boldsymbol{d}_{i}+$ $l_{i}^{2} \geq 0$. If it is not the case, it means that the distance between the platform joint and the corresponding base joint is greater than the link's length.

ii. $\quad q_{\min } \leq q_{i} \leq q_{\max }$ : for the joint coordinates an upper-bound and a lower-bound are imposed. If one or more of the joint coordinates were out of this variation range, the manipulator would approach a singularity condition.

iii. $\theta_{A, i} \leq \theta_{A, \text { lim }}$ and $\theta_{B, i} \leq \theta_{B, \text { lim }}$ : each joint must respect the mobility range given by the constructor. The angular shift is computed with respect to the condition in which the TCP is coincident with the center of the WSd with null orientation.

II. Kinetostatic constraint: If for hypothesis a set of unitary forces and moments is applied to the TCP the maximum force transmitted to the motors for a prescribed pose is computed as:

$\boldsymbol{\tau}_{\text {max }}(\boldsymbol{X})=\left\|J_{n}^{T}(\boldsymbol{X})\right\|_{\infty}$

The kinetostatic constraint is respected if the computed value is lower or equal to the imposed limit value $\tau_{\max , \mathrm{lim}}$.

III. Geometric constraints: The geometric constraints represent the respect of the minimum distance between the links and between each link and the mobile platform. The minimum distance between two elements is computed as the minimum distance between two segments in space. The platform is outlined as a $500 \mathrm{~mm}$ tall cylinder whose symmetry axis is orthogonal to the plane where the platform joint lie. For each pose it's necessary to assess that:

$\left\{\begin{array}{l}d_{\text {link }, i j} \geq d_{\text {link,min }} \quad \forall i, j \text { with } \quad i \neq j \\ d_{\text {link-platform }} \geq d_{\text {link-platform,min }} \quad \forall k\end{array}\right.$

\subsection{Machines parameterization}

Thanks to the symmetry that characterizes both the Gough-Stewart platform and the Hexaslide it is possible to use a four variable parametrization for the two manipulators.

The Gough-Stewart hexapod is made of a mobile platform on which three couples of spherical joints lie, each of them out of phase of $120^{\circ}$ with respect to the others. On the fixed base the disposition of the universal joints is analogous to that of the platform joints. The chosen variables used to parametrize the GS-platform are: (1) $R_{b}$, radius of the circumference the base joints $A_{i}$ lie on; (2) $\theta_{b}$, semi angular displacement between the two segments that connect the origin with a couple of base joints; (3) $R_{p}$, radius of the circumference the platform joints $B_{i}$ lie on; (4) $\theta_{p}$, semi angular displacement between the two segments that connect the TCP with a couple of platform joints. Usually the couples of platform joints are organized as $[(1,2)(3,4)(5,6)]$, while the couples of base joints are organized as $[(1,6)(2,3)(4,5)]$.

In the Hexaslide architecture the length of the links is fixed and equal for all the links. On the base there are three couples of parallel guides, each of them out of phase of $120^{\circ}$ with respect to the others about the $\mathrm{z}$ axis. For this architecture the parameterization variables are: (1) $s$, distance between two parallel guides; (2) $l_{\text {link }}$, length of the six links; (3) $R_{p}$, radius of the circumference the platform joints $B_{i}$ lie on; (4) $\theta_{p}$, semi angular displacement between the two segments that connect the TCP with a couple of platform joints.

\section{Numerical Results}

To optimize the machines an upper and a lower limits to the parameterization variables must be imposed. As told before one of the most critical aspects of PKMs is represented by the mobility range of the joints. Two kinds of joints are taken into account: (1) the spherical joints by Hephaist Seiko with a mobility range of $\pm 25^{\circ}$; (2) common universal joints with a mobility range of $\pm 40^{\circ}$. Even if the formers present lower backlash problem with respect to the universal ones, they prevent to have an acceptable coverage of the workspace.

The strategy used for the optimization process are: (1) gradual reduction of $\tau_{\text {max, lim }}$ starting from a high value and decreasing it in each new optimization as long as the coverage of the workspace is acceptable. (2) reduction of the upper-bound limit of the links length. During the optimization process one can notice that final value of the links length always converges to the highest limit. Since this parameter gives an idea of the total size of the manipulator, it's desirable to reduce it as much as possible. For this reason, once the minimum value of $\tau_{\max , \text { lim }}$ is found, the next requirement is to reduce the upper limit of the links length as long as the workspace is entirely covered. 


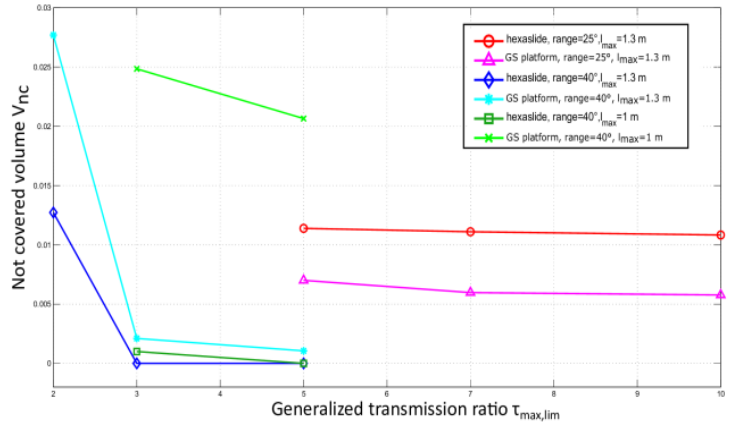

Figure 3. Not-covered volume for the two manipulators for various values of $\tau_{\max , \text { lim. }}$.

The comparison between the two machine can be seen Figure 3. It can be noticed that when using spherical joints the not covered volume is too high even if the value of $\tau_{\max \text {,lim }}$ is set to 10 . This means that in this case the limiting factor is represented by the joints mobility range. For this reason it's necessary to switch to the universal joints. If the parameter $l_{\max }$ is set to $1,3 \mathrm{~m}$ the Hexaslide allows to have a total coverage of the workspace with a $\tau_{\max \text {,lim }}$ value that is lower than 3 . However a 1,3 $\mathrm{m}$ long link is excessive and so, while still having a total coverage, the parameter $l_{\max }$ is lowered to $1 \mathrm{~m}$ accepting to have higher forces along the guides axes and consequently on the motors. On the other hand the Gough-Stewart is not able to guarantee a total coverage of the workspace even if the maximum link length is 1,3 $\mathrm{m}$. After these considerations the chosen machine is the Hexaslide whose schematic design is reported in Fig 4.
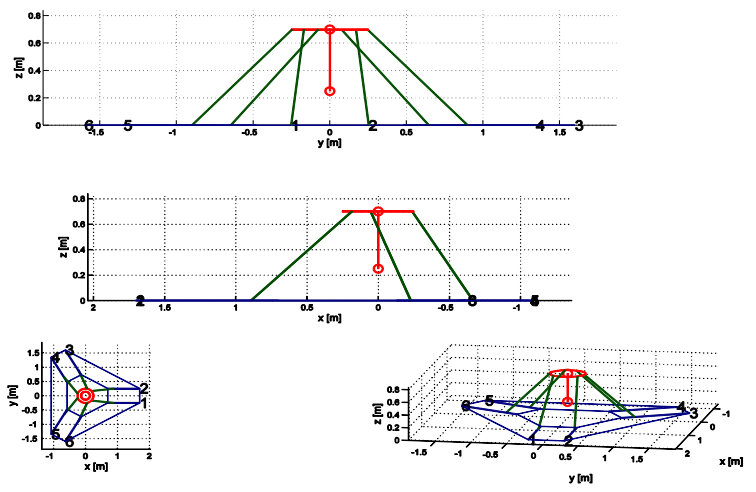

Figure 4. Hexaslide final structure.

\section{Conclusions}

This paper presents the optimization process of two parallel kinematic manipulators using a genetic algorithm given the technical specifications in terms of desired workspace for a particular application, that is the simulation of the sea waves through a mathematical model in wind tunnel tests. At the end of the optimization process it's clear that the only manipulator that is suitable for this specific task is the Hexaslide, a machine characterized by a high stiffness and a low inertia and that prevents the risk of collision between its elements. The optimization phase is the first and fundamental step of the machine synthesis process; as a matter of fact only once the dimensions and the characteristic parameters of the machine are well defined it will be possible to perform the following analyses.

For example it will be possible to built two dynamical models: (1) a rigid one used to size the drive system following the procedure described in $[10,11]$; (2) a flexible one to perform a vibration analysis in order to map the system's first natural frequency all over the workspace as described in $[12,13]$.

\section{References}

1. Bayati I., Belloli M., Ferrari D., Fossati F. \& Giberti H., "Design of a 6-DoF robotic platform for wind tunnel tests of floating wind turbines", Energy Procedia, pp. 313 (2014).

2. Merlet J. P., Parallel Robots, Springer, (2006).

3. Bonev I. A., Mechanism and Machine Theory, 36, 1 (2001).

4. Merlet J. P., Gosselin C., Handbook of Robotics , (2008).

5. Legnani G., Tosi D., Fassi I., Giberti H., Cinquemani S., "The "point of isotropy" and other properties of serial and parallel manipulators", Mechanism and Machine Theory, vol. 45, no. 10, pp. 1407-1423, (2010).

6. Legnani G., Fassi I., Giberti H., Cinquemani S., Tosi D., "A new isotropic and decoupled 6-DoF parallel manipulator", Mechanism and Machine Theory, vol. 58, pp. 64-81 (2012).

7. Fassi I., Legnani G., Tosi D. , "Geometrical conditions for the design of partial or full isotropic hexapods", Journal of Robotic Systems, vol. 22, no. 10, pp. 507-518, (2005).

8. Cinquemani S., Ferrari D., "A genetic algorithm optimization for IMSC technique", 2014 IEEE Conference on Control Applications, CCA 2014, pp. 1942.

9. Giberti H., Ferrari D., "A novel hardware-in-the-loop device for floating offshore wind turbines and sailing boats", Mechanism and Machine Theory, vol. 85, pp. 82-105, (2015).

10. Giberti H., Cinquemani S., Legnani G., "A practical approach to the selection of the motor-reducer unit in electric drive systems", Mechanics Based Design of Structures and Machines, vol. 39, no. 3, pp. 303-319, (2011).

11. Giberti H., Cinquemani S., Legnani G., "Effects of transmission mechanical characteristics on the choice of a motor-reducer", Mechatronics, vol. 20, no. 5, pp. 604-610, (2010).

12. Giberti H., Ferrari D., Fiore E., " Dynamics Modeling and Accuracy Evaluation of a 6-DoF Hexaslide Robot", Conference Proceedings of the Society for Experimental Mechanics Series, vol. 1, pp. 473, (2015).

13. Tarabini M., Roure A., Pinhede C. Active control of noise on the source side of a partition to increase its sound isolation (2009) Journal of Sound and Vibration, 320 (4-5), pp. 726-743 